\title{
Development of chronic colitis is dependent on the cytokine MIF
}

Ype P. de Jong et al.

In the November 2001 issue of Nature Immunology the name of an author was printed incorrectly. The name of ninth author listed should read Mazen Alsahli. 